\title{
Deuterium retention in tungsten films deposited by magnetron sputtering
}

\author{
P. Wang, W. Jacob*, L. Gao, S. Elgeti (Lindig), M. Balden \\ Max-Planck-Institut für Plasmaphysik, EURATOM Association, Boltzmannstr. 2, 85748 Garching, Germany
}

\begin{abstract}
:
Deuterium retention in tungsten films deposited on polycrystalline bulk tungsten substrates was investigated and compared with deuterium retention in $\mathrm{W}$ films deposited on silicon and in polycrystalline bulk $\mathrm{W}$ alone. The structure of the deposited films was investigated by X-ray diffraction and scanning electron microscopy combined with focused ion beam cutting. $\mathrm{D}$ retention after implantation was measured by nuclear reaction analysis and temperature programmed desorption (TPD). The W films deposited on bulk W show a typical columnar epitaxial growth. After D implantation, high densities of blisters with diameters of about hundred $\mu \mathrm{m}$ were formed. Interestingly, the blisters are located within the $\mathrm{W}$ substrate well below the interface of deposited film and substrate. TPD spectroscopy reveals two $\mathrm{D}_{2}$ release peaks at 510 and $700 \mathrm{~K}$, indicating at least two different trap energies.
\end{abstract}

Keywords: Tungsten Films, Microstructure, Deuterium retention, blistering, NRA, TPD

$\begin{array}{ll}\begin{array}{l}\text { Published in: } \\ \text { doi: }\end{array} & \begin{array}{l}\text { Physica Scripta T159 (2014) 014046 (6pp). } \\ 10.1088 / 0031-8949 / 2014 / \mathrm{T} 159 / 014046\end{array} \\ & \\ \text { Submitted: } & 10.05 .2013 \\ \text { Accepted: } & 24.09 .2013 \\ \text { Available online: } & 01.04 .2014\end{array}$

\footnotetext{
${ }^{*}$ Corresponding author. Fax:+49-3299-1504, Tel:+49-3299-2618.

E-mail address: wolfgang.jacob@ipp.mpg.de
} 


\section{Introduction}

Because retention of large quantities of tritium in the first-wall materials increases the fuel costs of a reactor and presents a safety concern, understanding of the fuel-retention properties of first-wall materials is important for ITER and next step fusion reactors such as DEMO [1-3]. Due to its favourable physical properties, such as low erosion yield and high melting temperature, tungsten is under consideration for use as plasma-facing material in the ITER divertor. Actually, a full tungsten inner wall and a full tungsten divertor have already been successfully developed and tested in ASDEX Upgrade and JET, respectively [4-7]. However, up to now mostly tungsten coatings were used as first-wall material for the so-called full-metal fusion devices, predominantly due to design limitations and cost concerns. Consequently, the influence of coating structure on the D retention should be taken into account.

Some results for $\mathrm{D}$ retention in $\mathrm{W}$ films used in ASDEX Upgrade were published by Ogorodnikova et al. [8]. We used W films deposited by magnetron sputtering as a model system to investigate the influence of film structure on the deuterium retention. Different film structures were achieved by using different deposition parameters or annealing treatments. Deuterium loading was performed in well-quantified deuterium plasma. In this work, D retention in $\mathrm{W}$ films deposited on polycrystalline bulk $\mathrm{W}$ was studied and compared with deuterium retention in $\mathrm{W}$ films deposited on silicon and in polycrystalline bulk $\mathrm{W}$ alone. $\mathrm{D}$ retention and release and blistering after D implantation were investigated and are discussed in this article.

\section{Experimental details}

Tungsten films with a thickness of $2.8 \mu \mathrm{m}$ were deposited using a commercial sputtering device (Discovery ${ }^{\circledR} 18$, Denton). The system was pumped down to a base pressure of less than $3 \times 10^{-5} \mathrm{~Pa}$. Deposition was performed in argon atmosphere at $0.5 \mathrm{~Pa}$. A liquid $\mathrm{N}_{2}$ cold trap was attached to the deposition chamber to reduce the water partial pressure. Prior to deposition the substrates were etched by argon plasma at $-600 \mathrm{~V}$ bias for 2 minutes to remove the native oxide layer. During $\mathrm{W}$ deposition the dc power applied to the tungsten sputter target was kept constant at $300 \mathrm{~W}$. At these settings the deposition rate of $\mathrm{W}$ is about $17 \mathrm{~nm} / \mathrm{min}$. Sample rotation during the deposition assured homogenous layer thickness. No extra substrate bias and heating or cooling was applied. During W film deposition the temperature of the substrate holder rises to about $370 \mathrm{~K}$. The sample temperature is estimated to be higher by at most $100 \mathrm{~K}$.

In this work, rolled $0.8 \mathrm{~mm}$ thick polycrystalline tungsten samples (manufactured by PLANSEE Metall GmbH, 6600 Reutte, Austria) with a guaranteed purity of $99.97 \mathrm{wt} . \%$ were used as substrates. Samples were mechanically polished to a mirror-like finish and cleaned in an ultrasonic bath with acetone. Prior to $\mathrm{W}$ film deposition the samples were stress-relieved and degassed by heating for 2 hours at $1200 \mathrm{~K}$ in an ultra-high vacuum oven ( $<<510^{-7} \mathrm{mbar}$ ). More details about the preparation of reference samples can be found in Ref. [9, 10]. For comparison, deuterium was also implanted in uncoated, plain polycrystalline $\mathrm{W}$ substrates. We note, that $\mathrm{W}$ films sputter-deposited in this device under identical conditions on silicon substrates had a density of about $90 \%$ of the $\mathrm{W}$ bulk density [14] and films deposited on bulk $\mathrm{W}$ substrates had a density of about $95 \%$. Appling ion beam analysis and x-ray photoelectron spectroscopy we found no traces of oxygen in the deposited layers. For details see [14]. Both, Rutherfors backscattering spectrometry (RBS) and temperature programmed desorption (TPD) were employed to investigate possible retention of Argon in the sputter-deposited layers. With RBS - which has a relatively low sensitivity (about 1\%) for Ar in W films - we found no indication of Ar. TPD resulted in an Argon amount comparable to 1 monolayer (about $1 \times 10^{15} \mathrm{~cm}^{-2}$ ). This amount of Ar could be implanted into the silicon or $\mathrm{W}$ substrates during 
the cleaning step prior to film deposition. In contrast, CMSII layer (as used by Ogorodnikova et al. [8]) show a clearly detectable Ar concentration of about $1 \%$.

The crystallographic phase formed in the $\mathrm{W}$ films was determined by X-ray diffraction (XRD) (Seifert XRD 3003 PTS) using a $\mathrm{Cu} \mathrm{K \alpha}$ source $(0.154 \mathrm{~nm})$. Diffractograms were acquired from 20 to $90^{\circ}$. The used scanning electron microscope (SEM) (Helios NanoLab 600, FEI) allows the cross-sectioning in situ by the implemented focused ion beam (FIB). The secondary electrons produced by a $5 \mathrm{keV}$ electron beam were detected by the inlens detector system. The cross sections were imaged with the e-beam tilted by $38^{\circ}$ to their surface plane, so that the vertical scale in the shown images is compressed relative to the horizontal scale.

Deuterium implantation was carried out in the laboratory plasma experiment PlaQ. A basic description of $\mathrm{PlaQ}$ is given in $[11,12]$. In short, $\mathrm{PlaQ}$ consists of a stainless steel chamber and is equipped with a remote electron cyclotron resonance (ECR) plasma source. Microwaves $(2.45 \mathrm{GHz})$ are coupled into the vacuum vessel through a waveguide terminated by a quartz window located at the high B-field side. The magnetic field is created by a single magnetic coil. To decouple the plasma from the substrate, the plasma is confined in a metallic cage of $150 \mathrm{~mm}$ in height and $140 \mathrm{~mm}$ in diameter. Particles can leave the cage in an axial direction through a hole in the bottom plate with a diameter of $55 \mathrm{~mm}$. A diverging plasma beam impinges perpendicularly onto the substrates which are located $100 \mathrm{~mm}$ below the cage exit. The energy of the ions impinging on the substrates was controlled by applying a dc bias to the substrate electrode.

To ensure identical conditions in all implantation processes, the microwave output power for the ECR plasma was set to $144 \mathrm{~W}$ with a constant $\mathrm{D}_{2}$ gas pressure prior to plasma ignition of $1.0 \mathrm{~Pa}$ (gas flow $50 \mathrm{sccm}$ ). The total deuteron flux in the form of ions consists dominantly of $\mathrm{D}_{3}{ }^{+}$ions ( $94 \%$ of the impinging ions corresponding to $97 \%$ of the impinging deuterons in form of ions) with minor contributions of $\mathrm{D}_{2}^{+}\left(2 \%\right.$ of the deuterons) and $\mathrm{D}^{+}(1 \%$ of the deuterons) [12]. In this article we refer to the energy per deuterium atom of the dominant molecular ion species as the ion energy. For the implantation with a dc substrate bias of $-100 \mathrm{~V}$ this results together with the plasma potential of about $-15 \mathrm{~V}$ [12] in an ion energy of about $115 \mathrm{eV}$, corresponding to a mean energy of about $38 \mathrm{eV}$ per deuteron for the dominant $\mathrm{D}_{3}{ }^{+}$ions. The total deuteron flux for these settings is $9 \times 10^{19} \mathrm{Dm}^{-2} \mathrm{~s}^{-1}$ [12]. In the experiment described here deuterium implantation was carried out at a sample temperature of $370 \mathrm{~K}$. The temperature of the sample holder is stabilized using an open circuit thermostat with silicon oil. Temperatures are measured by a thermocouple attached to the sample holder from the back side and by an infrared camera observing the sample surface. More details about sample temperature control can be found in Refs. [12,14].

The amount of $\mathrm{D}$ retained in the samples was measured by nuclear reaction analysis (NRA) two months after implantation using the $\mathrm{D}\left({ }^{3} \mathrm{He}, \alpha\right)$ p nuclear reaction. In the meantime samples were stored in a desiccator. The D concentration within the near-surface layer (at depths up to about $0.3 \mu \mathrm{m}$ ) was determined at a ${ }^{3} \mathrm{He}$ energy of $0.69 \mathrm{MeV}$ by analyzing the emitted $\alpha$ particles with a surface barrier detector at scattering angle of $102^{\circ}$. For determining the D concentration at larger depths, the energy of the analyzing beam of ${ }^{3} \mathrm{He}$ ions was varied from 0.69 to $4.5 \mathrm{MeV}$ depending on the thickness of $\mathrm{W}$ layer. The high energy protons from the $\mathrm{D}\left({ }^{3} \mathrm{He}, \mathrm{p}\right)^{4} \mathrm{He}$ nuclear reaction were counted using a solid state detector at a scattering angle of $135^{\circ}$ equipped with a curved slit reducing the solid angle to $29.9 \mathrm{msr}$. In order to analyze the D concentration profile in the whole layer NRADC [13] was used for the deconvolution of the NRA spectra measured at different ${ }^{3} \mathrm{He}$ ion energies. Details about the data evaluation using NRADC can be found in Ref. $[13,14]$. For the quantitative analysis we used the cross section published by Alimov et al. [15].

After the NRA measurement the retained D amount was additionally measured by TPD in the quartz tube of the TESS device. A basic description of TESS is given in [16]. The samples were heated up to a sample temperature of $1275 \mathrm{~K}$ with an oven heating rate of $15 \mathrm{~K} / \mathrm{min}$ [14]. 
The desorbed gases were measured with a quadrupole mass spectrometer (QMS). For quantitative analysis the QMS signals for $\mathrm{HD}$ and $\mathrm{D}_{2}$ were calibrated, and the calibration factors are $(3.5 \pm 0.14) \times 10^{9}$ molecules per count, and $(3.8 \pm 0.15) \times 10^{9}$ molecules per count, respectively.

\section{Results and discussion}

\subsection{Microstructures of magnetron-sputtered $W$ films}

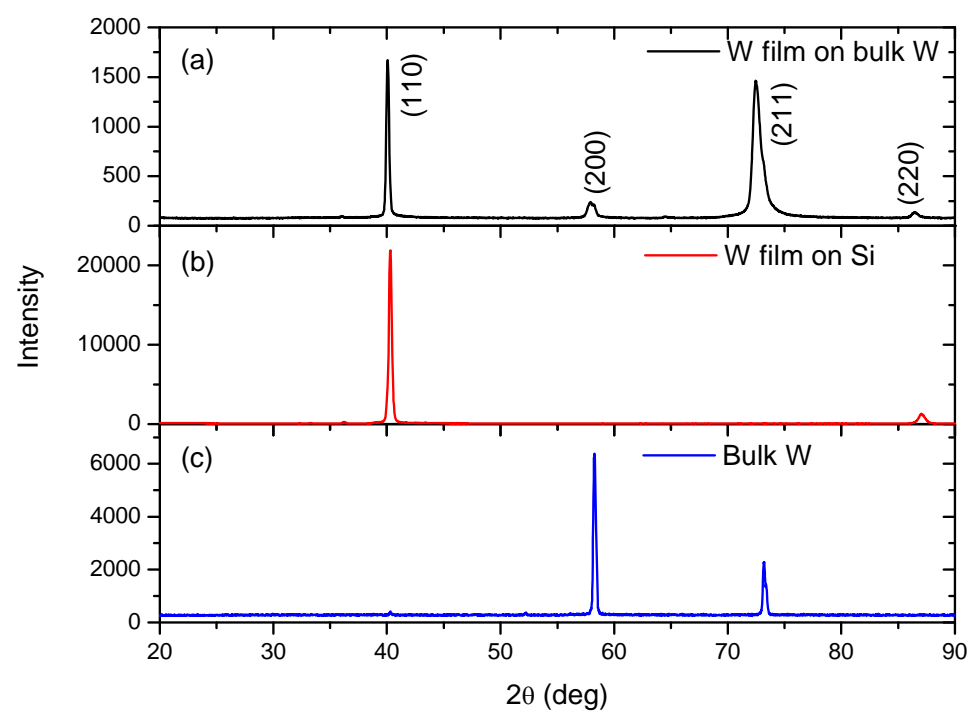

Fig. 1: X-ray diffractogram of W film deposited on bulk tungsten (a) and Si (b). The XRD diffractogram of hot-rolled bulk $W(c)$ is also shown for comparison.

The X-ray diffractograms of W films deposited on bulk W and Si are shown in Fig. 1a and $\mathrm{b}$, respectively. The $\mathrm{W}$ film deposited on bulk W (Fig. 1a) shows three diffraction peaks located at $40.0,58.0$ and $72.7^{\circ}$. These are typical diffraction peaks present in $\alpha$-phase $\mathrm{W}$. They correspond to the (110), (200), and (211) crystal orientation, respectively. All peak positions are shifted to lower diffraction angles by about 0.2 to $0.4^{\circ}$ compared with the standard powder diffractogram of $\mathrm{W}$ powder. This indicates that the crystal plane spacing in as-deposited $\mathrm{W}$ films is expanded, which could be due to the residual tensile stress within the $\mathrm{W}$ films accumulated during deposition. An apparent feature of the $\mathrm{W}$ film deposited on $\mathrm{Si}$ (Fig. 1b) is that the spectrum shows one strong and sharp peak at $40.3^{\circ}$ and another weak peak at $87.0^{\circ}$, which correspond to the (110) and (220) orientation of $\alpha$-phase W. The other two orientations (200) and (211) present in typical $\alpha$-phase $\mathrm{W}$ are missing in $\mathrm{W}$ films deposited on $\mathrm{Si}$, indicating that a strong orientation texture has developed during deposition. The polycrystalline bulk $\mathrm{W}$ which is used as the substrate and as reference in this work shows another texture. It exhibits one strong peak at $58.3^{\circ}$ and a smaller one at $73.2^{\circ}$ (Fig. 1c), corresponding to the (200) and (211) orientation. Obviously, due to the hot-rolling manufacturing process the grains with (100) orientations are dramatically suppressed.

A cross-section image of the $\mathrm{W}$ film deposited onto bulk $\mathrm{W}$ is presented in Fig. 2. Two different grain features can be distinguished from this cross-section image. The lower part of the image shows the bulk W substrate, in which grain boundaries are oriented predominantly parallel to the sample surface. There, all grains show an elongation in the rolling direction and the average length of the grains is about 0.5 to $5 \mu \mathrm{m}$. At the interface between the $\mathrm{W}$ film (upper part) and the substrate (lower part), indicated by the short horizontal dashed line on the 
left side of the figure, three elongated grains are discernible below that line. One of these grains is outlined by a dashed line in Fig. 2. These three grains belong to the $\mathrm{W}$ substrate and show an irregularly shaped bottom as other grains in the $\mathrm{W}$ bulk and a flat upper surface which is produced by the mechanical polishing during substrate pre-treatment. Above this flat interface the about $2.8 \mu \mathrm{m}$ thick $\mathrm{W}$ film is visible. Obviously, the growth of this $\mathrm{W}$ film is strongly affected by the grains of the underlying $\mathrm{W}$ substrate and we find a typical epitaxial growth on the grains of the substrate. This is expected because the film and bulk material are identical so that the lattice parameters match. Initially, each growing $\mathrm{W}$ grain has similar lateral dimension as the corresponding underlying grain. During growth different grains have different growth rates in different crystal orientations leading to a slight roughening of the surface. In some cases, new grains nucleate within the growing film. Within the $\mathrm{W}$ film the grain boundaries are oriented preferably in the direction normal to the substrate surface.

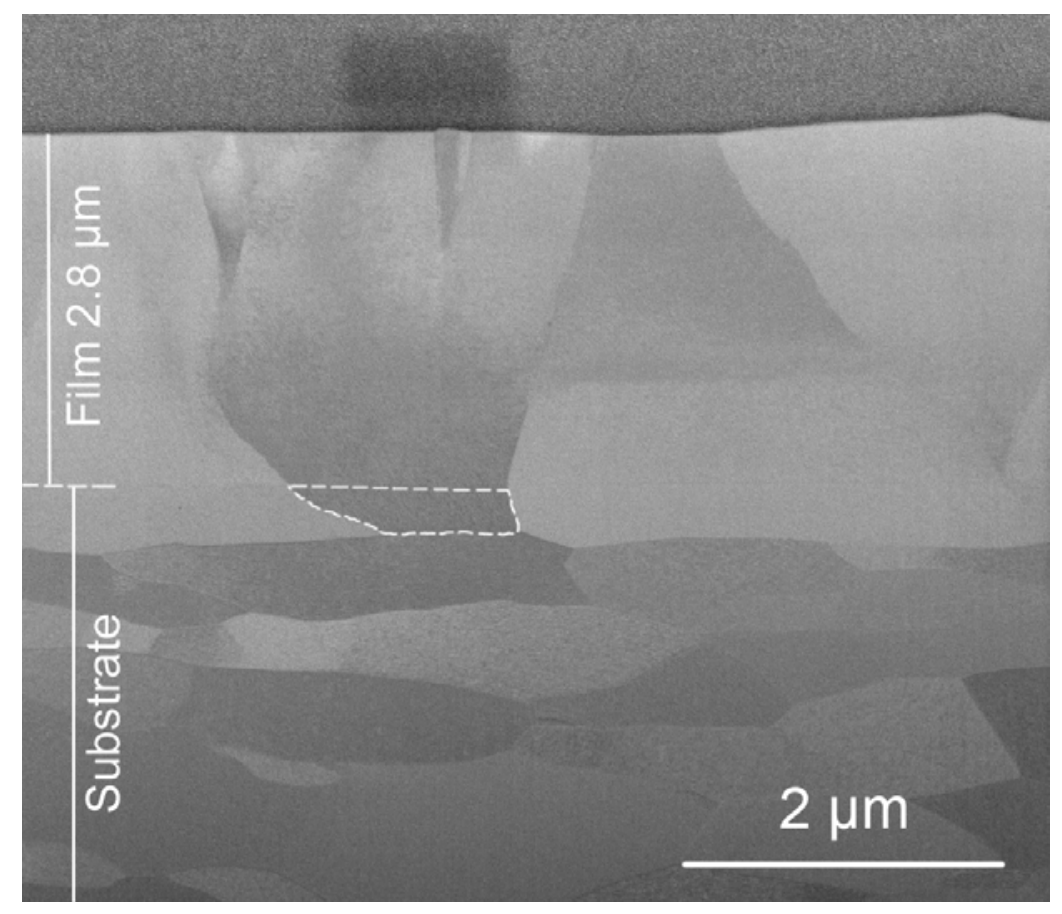

Fig. 2: Cross-section image of W film deposited on a hot-rolled, polycrystalline bulk W sample. The interface between the film and substrate is indicated by the short horizontal line at the left side of the figure. One grain from the top of the substrate is outlined by a dashed line. Note that the angle between the electron beam and the cross-section plane is $38^{\circ}$ so that the vertical distances appear in the image shorter that they are in reality.

\subsection{Blistering behaviour}

After deposition, some $\mathrm{W}$ films and a reference bulk $\mathrm{W}$ sample were simultaneously exposed to a deuterium plasma at $370 \mathrm{~K}$ substrate temperature with $38 \mathrm{eV} / \mathrm{D}$ incident energy and up to a fluence of $6 \times 10^{24} \mathrm{D} / \mathrm{m}^{2}$. After implantation, SEM and FIB cross-sectioning were used to collect information on blistering. The D retention was measured by both NRA and TPD. 

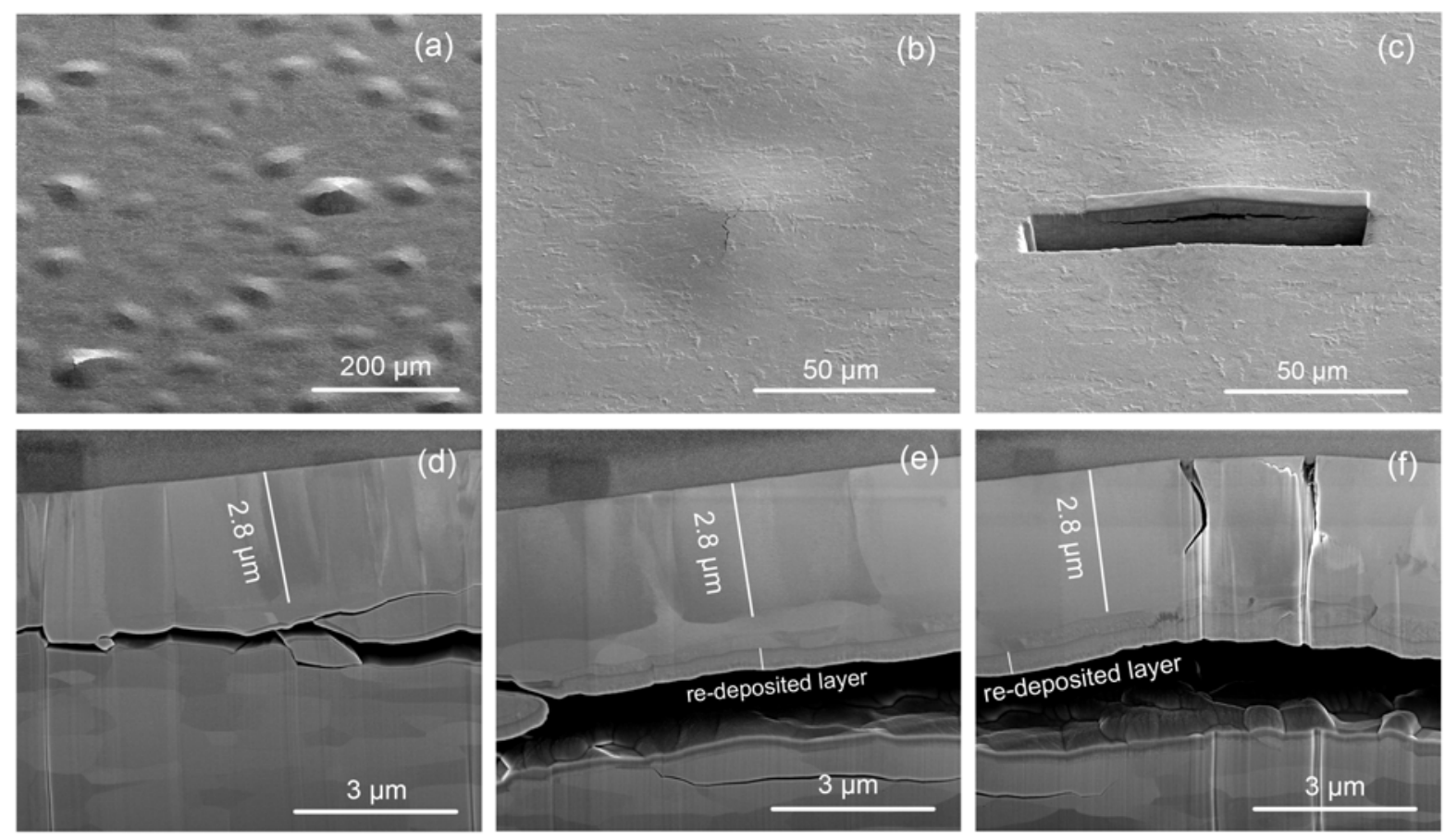

Fig. 3: Surface $\left(a, b, c\right.$, tilt angle $\left.52^{\circ}\right)$ and cross-section ( $\left.d, e, f\right)$ images of blisters formed after $\mathrm{D}$ implantation with $38 \mathrm{eV} / \mathrm{D}$ incident energy and a fluence of $6 \times 1024 \mathrm{D} / \mathrm{m} 2$ at $370 \mathrm{~K}$ substrate temperature. The thickness of the $W$ film and the re-deposited layer formed inside the cavity during FIB ion-beam cutting are marked in the cross-section images. In Fig. 3d the re-deposited layer was not marked because it is too thin. Note that the angle between the electron beam and the cross-section plane is $38^{\circ}$.

A high density of blisters was detected after D implantation on the surface of the W film deposited on bulk W (Fig. 3a). The size of these blisters varies from several tens up to nearly hundred micrometers, which is significantly larger than the blister formed in polycrystalline $\mathrm{W}$ implanted under the same conditions $[8,17,18]$. Some blisters are broken and the cracks are visible at the surface. One selected cracked blister (surface view in Fig. 3b) was cut by FIB and the full cross-section image is shown in Fig. 3c. It is obvious that the deformation due to blistering does not recover (at least not fully) after FIB cutting. The blister shows a crack system running parallel to the surface. Enlarged images of this blister focused on different regions of the cross-section are presented in Figs. 3d-f. In these three subfigures the thickness of the $(2.8 \mu \mathrm{m})$ deposited $\mathrm{W}$ film is marked. As in Fig. 2, the structure of the deposited film can be clearly distinguished from that of the underlying bulk $\mathrm{W}$ substrate. In the $\mathrm{W}$ film the grain boundaries are preferably oriented perpendicular to the surface while in the hot-rolled bulk tungsten substrate they are preferably oriented parallel to the surface. A continuous layer which is clearly visible at the upper side of the cavity in Figs. 3e and $\mathrm{f}$ is not from the original material, but it is due to redeposition of sputtered material during the FIB-cutting processes. These layers are marked in Figs. $3 \mathrm{e}$ and $\mathrm{f}$. They are about 0.3 and $0.4 \mu \mathrm{m}$ thick, respectively. In Fig. 3d the redeposition layer is not visible at the edge of the crack because the gap is too narrow. Apparently, the blister shown in Fig. 3 is not located within the $2.8 \mu \mathrm{m}$ thick $\mathrm{W}$ film, but occurs inside the bulk tungsten material. The topmost grains of the substrate which are elongated in direction parallel to the surface are clearly discernable on the lower side of the blister cap. We could not detect any indication of a crack between the deposited film and the substrate. In Fig. 3f two cracks can be seen in the central part of cross-section image. The white vertical lines in Fig. $3 \mathrm{f}$ are artefacts that result from the FIB cutting. The thickness of the material above the cavity is about $3.6 \mu \mathrm{m}$, which is thicker than the thickness of $\mathrm{W}$ film. In addition to the blister with cracks on the surface shown in Fig. 3, a blister without crack 
was studied by FIB cutting (results not shown here). In that case we also found a crack system parallel to the surface and located below the interface. This together with the cross-section images in Figs. 3d-f clearly demonstrates that the two blisters that were investigated in detail are neither located within the $\mathrm{W}$ film nor at the interface between film and substrate, but they occur at the first grain boundary inside the W substrate.

\subsection{D retention measured by NRA and TPD}

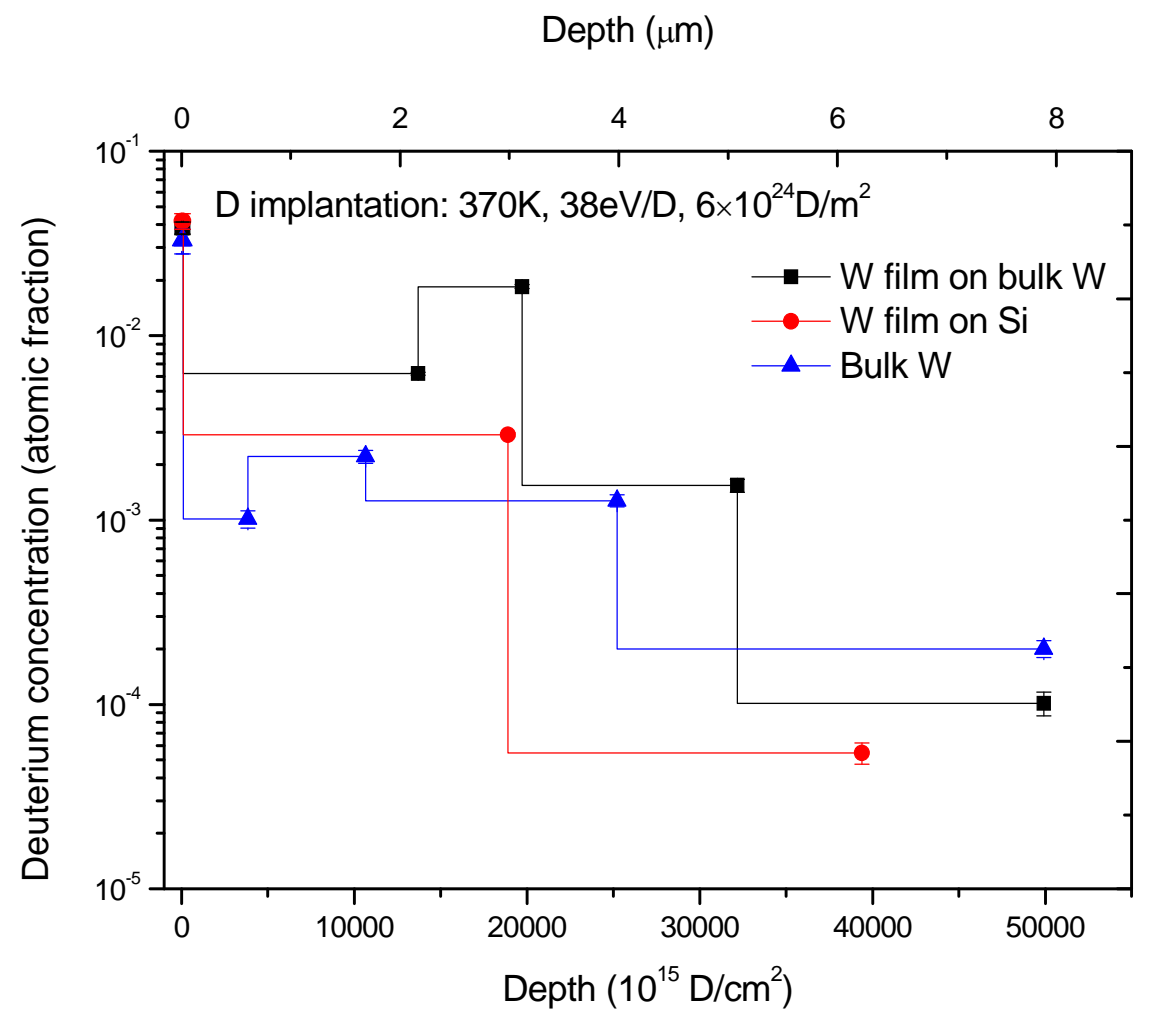

Fig. 4: Deuterium concentration depth profiles of W films deposited on bulk $W$ and Si. The D depth profile of bulk $W$ is also shown for comparison. All samples were implanted simultaneously with D at $370 \mathrm{~K}$ with $38 \mathrm{eV} / \mathrm{D}$ incident energy with a fluence of $6 \times 1024 \mathrm{D} / \mathrm{m} 2$.

Fig. 4 shows the D concentration depth profiles determined by NRA using ${ }^{3} \mathrm{He}^{+}$as a probing beam. For all samples implanted at $38 \mathrm{eV} / \mathrm{D}$ incident energy a high deuterium concentration is found in a thin near-surface layer with 3-4 \% D concentration. The thickness of this topmost layer is determined by the depth resolution of the $\alpha$ spectrum measured at $0.69 \mathrm{MeV}$ which is about $16 \mathrm{~nm}$. The given $\mathrm{D}$ concentration of $3-4 \%$ is the mean $\mathrm{D}$ concentration in this layer. More details regarding this issue can be found in Ref. [14]. Below this D-rich layer D retention shows different behaviours in the different investigated W materials. For the $\mathrm{W}$ film deposited on bulk W, D distributes homogenously to a depth of about $2 \mu \mathrm{m}$ with an atomic fraction of $6 \times 10^{-3}$. At the interface to the bulk W substrate a zone with slightly higher D concentration of about $2 \times 10^{-2}$ is found. Because the FIB cross-section images (Fig. 3) revealed that the blisters are formed in the vicinity of the interface, this D-enriched region could be attributed to the $\mathrm{D}$ retained inside the blister and in the surrounding. Comparable implantation experiments performed by Manhard [17] using the same $\mathrm{W}$ materials, also showed a high $\mathrm{D}$ concentration zone related to blisters being present in a depth range between 0.5 to $5 \mu \mathrm{m}$. Below the $\mathrm{W}$ film the $\mathrm{D}$ concentration in the bulk W drops to $1 \times 10^{-3}$ and reduces to $1 \times 10^{-4}$ in larger depth. In the W film deposited on Si the D profile is flat with an atomic fraction of about $3 \times 10^{-3}$. Beyond the depth corresponding to the 
film thickness the D concentration inside the silicon substrate drops by two orders of magnitude to $10^{-5}$, which is equivalent to the sensitivity limit of the analysis method in this case. In the bulk W sample without W film D distributes inhomogeneously in depth. In the depth range up to about $4 \mu \mathrm{m}$ the $\mathrm{D}$ concentration in bulk $\mathrm{W}$ is about $1-2 \times 10^{-3}$ and for larger depths the concentration drops by one order of magnitude to $10^{-4}$. The results for bulk tungsten are in excellent agreement with those from Ref. [17]. Obviously, D concentration in the $\mathrm{W}$ film on $\mathrm{W}$ substrate is with about $6 \times 10^{-3}$ significantly higher than in the reference bulk $\mathrm{W}\left(1-2 \times 10^{-3}\right)$. This could be due to a higher defect density in the $\mathrm{W}$ films compared with hot rolled polycrystalline bulk $\mathrm{W}$.

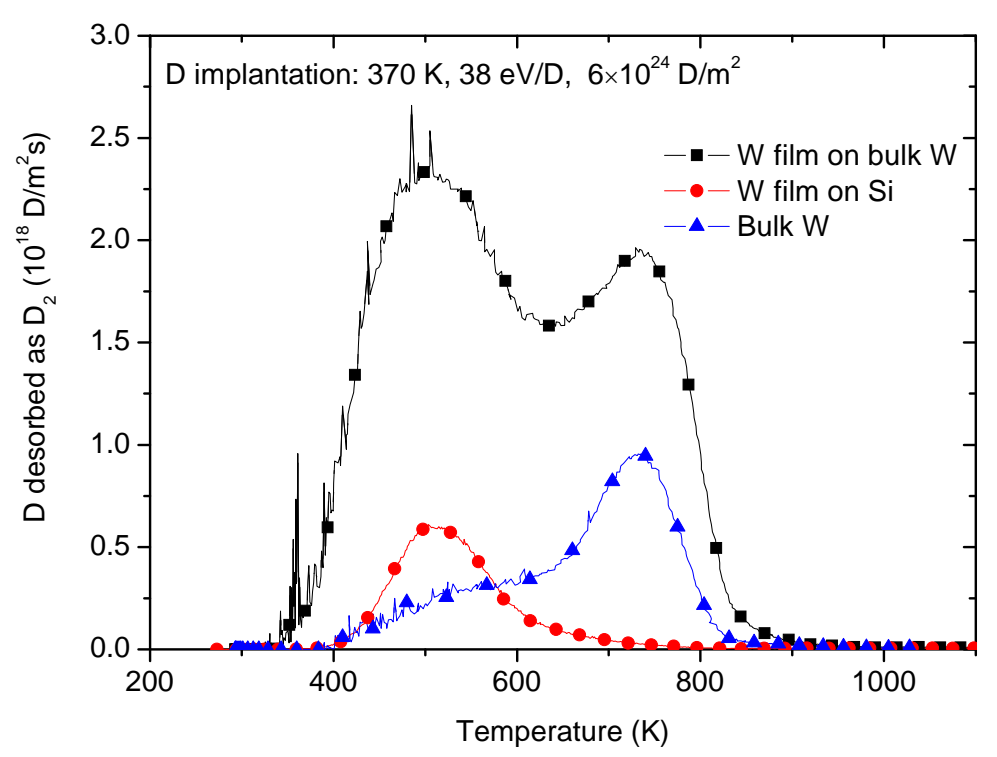

Fig. 5: D2 release spectrum from W films deposited on bulk $W$ and Si. For comparison the D2 release spectrum of bulk $W$ is also shown. In all cases, the oven heating rate is $15 \mathrm{~K} / \mathrm{min}$ (the real sample heating rate is slightly higher, 18 to $20 \mathrm{~K} / \mathrm{min}$ in the region of the peaks, see Ref. [14]).

After the NRA measurement the retained D amount was also measured by TPD. Fig. 5 shows the $\mathrm{D}_{2}$ release peaks of these three samples as a function of the sample temperature. HD release was also investigated, but is not shown. For all investigated W films the contribution of the HD peak to the total D release is at most $30 \%$. Some more details can be found in Ref. [14]. In these three spectra only two $\mathrm{D}_{2}$ release peaks at 510 and $730 \mathrm{~K}$ (oven heating ramp $15 \mathrm{~K} / \mathrm{min}$ resulting in sample heating rates of about 18 to $20 \mathrm{~K} / \mathrm{min}$ in the region of the peaks, see [14]) are detected. The intensities of these $\mathrm{D}_{2}$ release peaks are different for the different investigated $\mathrm{W}$ materials. For the $\mathrm{W}$ film deposited on $\mathrm{Si}$, only the release peak at $510 \mathrm{~K}$ occurs. In addition, the spectrum shows a tail extending to about $700 \mathrm{~K}$. The reference polycrystalline bulk $\mathrm{W}$ shows the main $\mathrm{D}_{2}$ release peak at $730 \mathrm{~K}$ and a shoulder peak at around $500 \mathrm{~K}$. For the $\mathrm{W}$ film deposited on bulk $\mathrm{W}$ the spectrum shows two $\mathrm{D}_{2}$ release peaks located at 510 and $730 \mathrm{~K}$, respectively. The appearance of two desorption peaks suggests the presence of at least two trap energies. These two desorption peaks are in excellent agreement with experiments performed under identical implantation conditions using polycrystalline $\mathrm{W}$ and applying the same heating ramp in TPD $[8,17,18]$. They are typical $\mathrm{D}_{2}$ release peaks reported for polycrystalline $\mathrm{W}$ or $\mathrm{W}$ films [19-21]. They are assigned to deuterium trapped in intrinsic defects such as grain boundaries and dislocations.

In addition, the spectra for bulk $\mathrm{W}$ and the $\mathrm{W}$ film on the $\mathrm{W}$ substrates shows in the low temperature part a slightly 'noisy' appearance. It has been shown before that the spikes 
occurring in this temperature range are due to the bursts of gas-filled blisters [18]. In bulk W implanted at the same condition these spikes are generally very weak but detectable at the heating ramp of $15 \mathrm{~K} / \mathrm{min}$. For TPD experiments applying slower heating ramps these spikes are much stronger [18]. However, for the $\mathrm{W}$ film deposited on bulk $\mathrm{W}$ these spikes are clearly detectable even at the oven heating ramp of $15 \mathrm{~K} / \mathrm{min}$. This indicates that in the latter case more $\mathrm{D}_{2}$ gas is released from individual blister bursts. This interpretation is in agreement with the fact that the blisters in the present case are significantly larger than the blister formed in polycrystalline $\mathrm{W}$ implanted under the same conditions $[8,17,18]$ (see Sect. 3.2).

\section{Conclusions}

Tungsten films deposited by magnetron sputtering were used as a model system to study the influence of the film microstructure on the deuterium retention behaviour. Both TPD and NRA results prove that deuterium retention in $W$ coatings strongly depends on the microstructures of the $\mathrm{W}$ films. The $\mathrm{W}$ film deposited on Si shows a flat deuterium depth profile with D atomic fraction of about $3 \times 10^{-3}$, no blisters were detected after D implantation. The $\mathrm{W}$ film deposited on polycrystalline bulk $\mathrm{W}$ shows a typical columnar epitaxial growth with grain boundaries perpendicular to substrate surface. D distributes in it with an atomic fraction of about $6 \times 10^{-3}$, which is by a factor of 3 higher than in bulk tungsten. A high density of blisters with diameters of several 10 up to about hundred micrometres was formed after D implantation. Corresponding SEM images after FIB cross-sectioning reveal that the cavities of these investigated blisters are located within the $\mathrm{W}$ substrate and occur at the bottom of the first layer of grain boundaries. The corresponding TPD spectrum reveals two $\mathrm{D}_{2}$ release peaks at 510 and $730 \mathrm{~K}$. The same release temperatures are found for polycrystalline bulk W and W films deposited on silicon.

\section{Acknowledgments}

The stay of P. Wang at Max-Planck Institute for Plasma Physics in Garching was funded through a bilateral agreement between Max-Planck Society and the Chinese Academy of Sciences which is gratefully acknowledged. One of the authors (L. Gao) is grateful to the finical support from MPG-CAS (Max-Planck Gesellschaft \& Chinese Academy of Sciences) Joint Doctoral Promotion Programme. 


\section{References}

[1] J. Roth, E. Tsitrone, A. Loarte, et al., J. Nucl. Mater., 390-391 (2009) 1-9.

[2] J. Roth, K. Schmid, Phys. Scr. T145 (2011) 014031.

[3] R. A. Causey, J. Nucl. Mater. 300 (2002) 91.

[4] O. Gruber, A. C. C. Sips, R. Dux, et al., Nucl. Fusion. 49 (2009) 115014.

[5] M. Kaufmann, R. Neu, Fusion Eng. Des. 82 (2007) 521.

[6] T. Hirai, H. Maier, M. Rubel, Ph. Mertens, et al., Fusion Eng. Des. 82 (2007) 1839.

[7] H. Maier, J. Luthin, M. Balden, S. Lindig, et al., J. Nucl. Mater. 307-311 (2002) 116.

[8] O.V. Ogorodnikova, K. Sugiyama, T. Schwarz-Selinger, T. Dürbeck, M. Balden, J. Nucl. Mater. 419 (2011) 194.

[9] A. Manhard, K. Schmid, M. Balden, W. Jacob, J. Nucl. Mater. 415 (2011) S632.

[10] A. Manhard, G. Matern, M. Balden, Practical Metallography, 50 (2013) 5.

[11] T. Schwarz-Selinger, A. von Keudell, and W. Jacob, J. Appl. Phys. 86, (1999) 3988.

[12] A. Manhard, T. Schwarz-Selinger, W. Jacob, Plasma Sour. Sci. Technol. 20 (2011) 015010. Note: Unfortunately, the information given in the last paragraph of this article is not correct, but the information in figures 5 and 6 is correct. The contribution of the molecular ions to the total ion flux for standard conditions is: $\mathrm{D}_{3}{ }^{+}=94 \%, \mathrm{D}_{2}{ }^{+}=3 \%$ and $\mathrm{D}^{+}=3 \%$. Correspondingly, the contributions to the total deuteron flux in form of ions are: $97 \%, 2 \%$, and $1 \%$.

[13] K. Schmid, U. von Toussaint, Nucl. Instr. Meth. B 281 (2012) 64.

[14] P. Wang, W. Jacob, L. Gao, T. Dürbeck, T. Schwarz-Selinger, Nucl. Instr. Meth. B 300 (2013) 54.

[15] V. Kh. Alimov, M. Mayer, J. Roth, Nucl. Instr. Meth. B 234 (2005) 169.

[16] E. Salançon, T. Dürbeck, T. Schwarz-Selinger, F. Genoese, W. Jacob, J. Nucl. Mater. 376 (2008) 160.

[17] A. Manhard, Deterium inventory in tungsten after plasma exposure: A microstructural survey. Ph.D thesis, University Augsburg 2012, also available as IPP Report Number: $17 / 34$.

[18] A. Manhard, U. von Toussaint, T. Dürbeck, K. Schmid, W. Jacob, Phys. Scr. T145 (2011) 014038.

[19] W. M. Shu, E. Wakai, T. Yamanishi, Nucl. Fusion. 47 (2007) 201.

[20] O. V. Ogorodnikova, J. Roth, M. Mayer, J. Appl. Phys. 103, (2008) 034902.

[21] V. Kh. Alimov, J. Roth, W. M. Shu, D. A. Komarov, K. Isobe, T. Yamanishi, J. Nucl. Mater. 399 (2010) 225. 